\title{
Complicated clinical course of acute pelvic inflammatory disease
}

\author{
Anna Kornete*, Zane Paunina, Zane Grabe
}

Department of Obstetrics and Gynecology, Riga East Clinical University Hospital, Riga, Latvia

Received: 12 July 2017

Accepted: 08 August 2017

\section{*Correspondence:}

Dr. Anna Kornete,

E-mail: annakornete@gmail.com

Copyright: (c) the author(s), publisher and licensee Medip Academy. This is an open-access article distributed under the terms of the Creative Commons Attribution Non-Commercial License, which permits unrestricted non-commercial use, distribution, and reproduction in any medium, provided the original work is properly cited.

\section{ABSTRACT}

The absence of both the classic risk factors for pelvic inflammatory disease (PID) and the triad of cervical motion, uterine or adnexal tenderness does not preclude the diagnosis of PID and tubo-ovarian abscess. The clinical case demonstration presents the perimenopausal woman with complicated clinical course of acute PID.

Keywords: Pelvic inflammatory disease, Sepsis, Multi-drug resistance

\section{INTRODUCTION}

Pelvic inflammatory disease (PID) is a common and serious complication of sexually transmitted diseases in young women, but is rarely diagnosed among perimenopausal women. PID comprises a spectrum of inflammatory disorders of female genital tract, including tubo-ovarian abscess (TOA). The exact incidence of PID in perimenopausal women is unknown; however, less than $2 \%$ of women with TOA formation are perimenopausal. ${ }^{1}$ Despite the rarity with which PID occurs in perimenopausal woman, consideration and early recognition of the diagnosis can be crucial.

\section{CASE REPORT}

A 52-year-old woman was admitted to the emergency department complaining of severe fatigue, malaise and edema, sharp stabbing pain in the left leg. This was accompanied by a month-long history of lower abdominal pain, edema in the left leg for 10 days, diarrhea and decreased urine output for six and two days, respectively. Seven days prior the patient had visited the gynecologist. During the visit, after five years of use, an intrauterine device (IUD) was evacuated. Besides, left side ovarian mass suspicious for malignancy was detected by ultrasound. The patient's last menstrual period was six month ago; G2P0. The patient has been sexually active with one male partner for the past several years. The patient had no significant medical history other than morbid obesity grade 3 (BMI $43 \mathrm{~kg} / \mathrm{m}^{2}$ ).

Her vital signs on admission showed a temperature of $36.8^{\circ} \mathrm{C}$, a blood pressure of $100 / 57 \mathrm{~mm} \mathrm{Hg}$, a heart rate of 96 beats per minute and a respiratory rate of 16 times per minute. During clinical examination, the patient's condition was serious, though there were no alteration in mental status.

The left thigh was severely enlarged and painful. Abdominal ultrasonography revealed acute renal parenchymal damage, left side hydronephrosis grade II. Gynecologic ultrasonography revealed tubo-ovarian abscess (TOA) on the left side with the size of the left ovary of $7.5 \times 5.5 \mathrm{~cm}$. Doppler ultrasonography imaging could not exclude deep venous thrombosis in the left leg.

Laboratory tests were as follows: white blood cell count $20.90 \times 10^{\wedge} 9 / \mathrm{L}\left(4.00-9.00 \times 10^{9} / \mathrm{L}\right)$ with $88.8 \%$ neutrophils (48.0-78.0\%), red blood cells $4.11 \times 10^{12} / \mathrm{L}$ (3.8-5.3 $\left.\mathrm{x} 10^{12} / \mathrm{L}\right)$, hemoglobin $9.74 \mathrm{~g} / \mathrm{dL}(12.00-14.00 \mathrm{~g} / \mathrm{dL})$, hematocrit $32.3 \%(35.00-45.00 \%)$, platelets $563 \times 10^{9} / \mathrm{L}$ $\left(150-400 \times 10^{9} / \mathrm{L}\right)$, C-reactive protein $327.15 \mathrm{mg} / \mathrm{L}(0-5$ $\mathrm{mg} / \mathrm{L})$, procalcitonin $5.23 \mathrm{ng} / \mathrm{mL}(0.00-0.05 \mathrm{ng} / \mathrm{mL})$, 
partial thromboplastin time $37.9 \mathrm{~s}(28-40 \mathrm{~s})$, fibrinogen $13.01 \mathrm{~g} / \mathrm{L} \quad(2-4 \mathrm{~g} / \mathrm{L})$, D-dimer $5.01 \mu \mathrm{g} / \mathrm{mL} \quad(0.00-0.50$ $\mu \mathrm{g} / \mathrm{mL})$, creatinine $314.00 \mu \mathrm{mol} / \mathrm{L}(44.00-80.00 \mu \mathrm{mol} / \mathrm{L})$, glomerular filtration rate $14.32 \mathrm{~mL} / \mathrm{min} / 1.73 \mathrm{~m}^{2}$ (kidney failure).

Considering clinical, laboratory and ultrasound imaging findings, the diagnosis of acute PID, bilateral salpingooophoritis, the left side TOA, sepsis, multiple organ dysfunction syndrome (MODS) was set.

Meanwhile, the patient was transferred to the intensive care unit and intravenous empirical antibiotic treatment with metronidazole and piperacillin/tazobactam was indicated. After initial doses with antibiotics, the patient's clinical condition severely worsened and she became hemodynamically unstable. The diagnosis of septic shock was made and therefore therapy with vasopressor agents was administered. Concerning surgical approach, the patient did not meet the criteria for a safe operative therapy, therefore it was decided to postpone operative therapy and assess the patient's condition in 12 hours.

The patient's condition remained severe, though within next hours she had become hemodynamically stable and there were no longer signs of septic shock, therefore vasopressor agents were cancelled. Within 18 hours of admission, the patient's medical condition improved. Midline laparotomy, subtotal hysterectomy with bilateral salpingo-oophorectomy, adhesiolysis and sanitation and drainage of abdominal cavity was performed. On surgery, bilateral TOAs and adhesions from the abscesses to the surround structures were found.

The test results for HIV and sexually transmitted diseases were negative. The blood and fecal cultures obtained on admission were negative. The cultures obtained from cervix and urine revealed E. coli, Bacillus spp., Corynebacterium spp., Candida spp. E. coli and $S$. constellatus were cultured from the pus from TOAs.

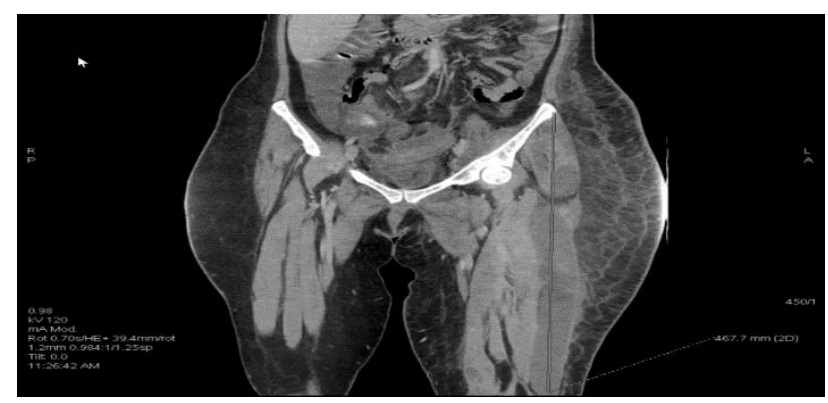

Figure 1: A CT scan of the abdominal and pelvic organs and both legs.

During the following postoperative period, the patient's condition improved, she was hemodynamically stable and diuresis was maintained. Though complaints about severe edema and pain in the left thigh remained. According to Doppler ultrasonography imaging deep venous thrombosis in the left leg could not have been excluded and low-molecular-weight heparin therapy was initiated consequently. Due to persistent pain in the left thigh, computed tomography (CT) of the abdominal and pelvic organs and both legs were indicated (Figure 1). A CT scan confirmed multiple retroperitoneal small pelvic abscesses, multiple abscesses in the left gluteal area with the spread to muscles and interfascial space of the left thigh (with the total size more than $50.0 \times 12.0 \times 16.0$ $\mathrm{cm})$, bilateral pneumonia.

Three days after the prior surgery, a decision to proceed to the left thigh and left gluteal area fasciotomy was passed. More than $1500 \mathrm{ml}$ of greyish pus was drained and sent for culture. The cultures obtained during fasciotomy was positive for $S$. sanguinis. Within the following days, repetitive aggressive surgical wound debridement, bandaging, irrigation and drainage was conducted (Figure 2 and Figure 3).

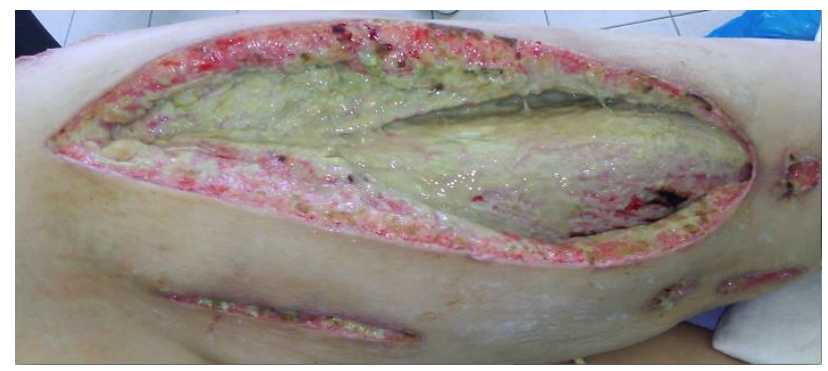

Figure 2: Post-operative wounds of the left thigh.

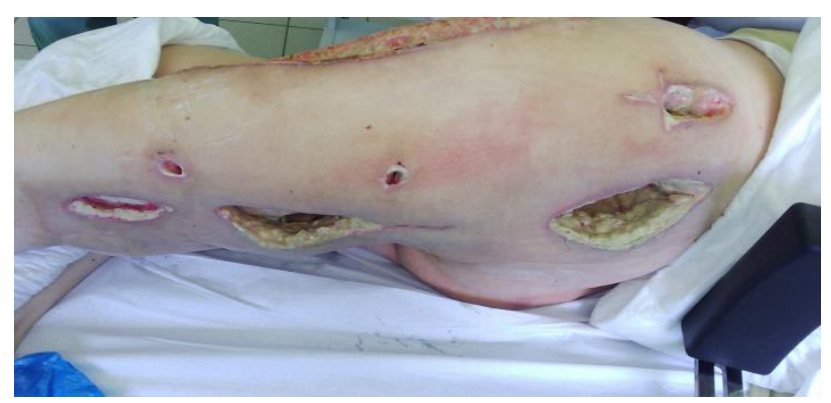

Figure 3: Post-operative wounds of the left thigh and gluteal area.

Seven days after fasciotomy, the patient's clinical condition aggravated severely and septic shock manifested. Therapy with vasopressor agents was initiated. Laboratory investigations demonstrated white blood cell count $13.20 \times 10^{9} / \mathrm{L}$ with $83.0 \%$ neutrophils, hemoglobin $10.6 \mathrm{~g} / \mathrm{dL}$, hematocrit $31.9 \%$, platelets 507 x $10^{9} / \mathrm{L}$, C-reactive protein $139.49 \mathrm{mg} / \mathrm{L}$, procalcitonin $0.404 \mathrm{ng} / \mathrm{mL}$. The blood cultures were positive for $S$. epidermidis. The urine culture was positive for multidrug resistant (MDR) A. baumannii and Candida spp. According to the results of antibiogram, antimicrobial therapy was switched to imipenem/cilastatin, vancomycin, flucanozole. On the $20^{\text {th }}$ day of hospitalization, in accordance with transesophageal echocardiography, the diagnosis of infective endocarditis 
was verified. The patient's operative risk was high, therefore watchful waiting was recommended.

Two weeks after fasciotomy, a magnetic resonance imaging (MRI) of the left thigh revealed reduction of the size of abscesses, no signs of new pus collections, large edema in gluteal and femoral muscles on the left side. The blood cultures were positive for MDR Staphylococcus epidermidis, MDR A. baumannii. The culture from wounds the thigh and gluteal area was positive for MDR A. baumannii.

After 26 days at hospital, the patient was transferred to clinic of surgical infection for subsequent wound debridement, bandaging and therapy with vacuumassisted closure system. During hospitalization, decubitus ulcers of the right gluteal area grade III developed.

On the $85^{\text {th }}$ day of hospitalization, the patient was discharged with the diagnosis: acute PID, bilateral salpingo-oophoritis, bilateral TOAs, multiple abscesses retroperitoneal in the small pelvis, left gluteal area and left thigh, necrotizing fasciitis (NF) in the left gluteal area and left thigh, sepsis, MODS, septic shock, bilateral pneumonia, infective endocarditis, decubitus ulcers of the right gluteal area grade III, morbid obesity grade 3 .

\section{DISCUSSION}

There is lack of most of common risk factors for PID in the patient in question, however, two risk factors could indicate the possible pathogenesis. The first, the vaginal flora of perimenopausal women is more likely to be colonized with aerobic gram-negative bacteria, especially E. coli, rather than sexually transmitted organism. Studies are showing, that E. coli is common in women with ruptured TOAs and a frequent cause of gram-negative sepsis. ${ }^{1,3}$ The second, use of an IUD has been linked to an increased risk of PID, but current IUDs may pose a substantially lower risk - less than $1 \% .{ }^{4}$ Some authors are saying that the use of an IUD for more than five years increases the risk of TOA. The authors also report that $E$. coli is the most common organism causing TOA among IUD users. ${ }^{5}$

TOAs, unlike other types of abscesses, tend to occur between organs rather than are confined inside an organ, therefore are more likely to spread locally ${ }^{1}$. The adherence of adjacent pelvic structures might serve a host defense mechanism to contain the inflammatory process within the pelvis. ${ }^{1}$ This could be a reason that some women with TOA have a vague course of the disease. Tubo-ovarian mass should be strongly considered as differential diagnosis in any IUD user who presents with complaints of abdominal pain, fever or adnexal mass. ${ }^{6,7}$ At the same time, malignancy should be a concern in any perimenopausal woman with an adnexal mass. ${ }^{6,7}$

As accurate preoperative discrimination between benign and malignant adnexal masses is known to be of pivotal value in clinical practice, it is suggested to implement the International Ovarian Tumor Analysis (IOTA) criteria. ${ }^{8}$ IOTA has published a consensus statement on terms, definitions and measurements to describe the sonographic features of adnexal masses, which is now widely used. CT is usually the imaging modality when the diagnosis is uncertain after ultrasound imaging; the concomitant involvement of the retroperitoneum and the ovary is depicted. ${ }^{2,9}$ However, MRI is the most precise tool to determine the location of origin of a pelvic mass, to characterize an adnexal mass, and to detect the local spread. ${ }^{2,9}$

TOA can also be associated with abscesses retroperitoneal in pelvis, gluteal area and thigh. ${ }^{11,12}$ As they are uncommon, they may be overlooked during radiologic examination and surgery worsening the patient's clinical outcome. Furthermore, these abscesses could progress to NF of the thigh subsequently. Being aware of many nonspecific ways abscesses can present in these locations, and having the patient with such vague clinical features, it could guide to an appropriate plan for the patient's management. ${ }^{13-15}$ Though one of the most severe complication of TAO may be NF. It is a rapidly progressive soft tissues infection that causes secondary necrosis of adjacent tissues. The characteristic features of NF are rapid tissue damage, systemic toxicity. Due to the nonspecific clinical presentations, diagnosis is usually delayed, or is confused with other clinical entities ${ }^{18}$. Importantly, in patients with necrotizing soft tissue infection, a delay of surgical treatment of more than 12 hours is associated with an increased number of surgical debridement and higher incidence of septic shock and acute kidney injurie. ${ }^{18}$

Early diagnosis of NF is crucial and is usually made when there is a high degree of clinical suspicion combined with radiologic examination. CT and MRI can be substantial in depicting early findings and assessing the extent of disease. ${ }^{17-19}$ Sepsis is defined as lifethreatening organ dysfunction caused by a dysregulated host response to infection and estimates indicate that sepsis is a leading cause of mortality and critical illness worldwide $^{20}$. For clinical practice, it is suggested to use a bedside clinical score-qSOFA criteria to identify septic patient. ${ }^{20}$ Notably, a negative blood culture is common and should not delay treatment as sepsis is a clinical diagnosis. ${ }^{14}$ Detrimental host responses to infection range from sepsis to septic shock and MODS.

In the literature, the paradox that antibiotic treatment of septic patients in some cases may actually elicit circulatory shock has been described. ${ }^{22,23}$ It has been suggested that the beta-lactam antibiotic-induced release of endotoxins is an important cause of the development of septic shock in patients treated for severe infections caused by gram-negative bacteria. ${ }^{22,23}$

Since antibiotic resistance is one of the most important problem worldwide, the control of the sepsis and septic 
shock should be done by a constant monitoring of the microbial involvement and antimicrobial susceptibility. Concerning this aspect, the main problem in the clinical case is the presence of MDR $S$. epidermidis and MDR $A$. baumannii leading to septic shock as a result of MDR organism induced sepsis. ${ }^{24,25}$

\section{CONCLUSION}

The clinical demonstration represents a challenging case of a perimenopausal woman with complicated clinical course of acute PID. Awareness with clinical and imaging findings of retroperitoneal involvement that may be found in association with PID and TOA could help to make an accurate diagnosis, thus facilitating patients' management. The clinical case emphasizes the severity of hospital acquired infection caused by MDR bacteria and possible difficulties in the guidance of these conditions.

Funding: No funding sources

Conflict of interest: None declared

Ethical approval: Not required

\section{REFERENCES}

1. Chappell CA. Pathogenesis, Diagnosis, and Management of Severe Pelvic Inflammatory Disease and Tuboovarian Abscess. Clin Obstet Gynecol. 2012;55:893-903.

2. Lareau SM, et al. Pelvic Inflammatory Disease and Tubo-ovarian Abscess. Infect Dis Clin N Am. 2008;22:693-708.

3. Jackson SL. Pelvic Inflammatory Disease in the Postmenopausal Woman. Ind Dis Obstet Gynecol. 1999;7:248-52.

4. Shepherd SM. Pelvic Inflammatory Disease. Available fromhttp://emedicine.medscape.com/article/256448overvi ew.2017.

5. Hui CK. Recurrent extended-spectrum betalactamase-producing Escherichia coli urinary tract infection due to an infected intrauterine device. Singapore Med J. 2014;55(2):28-30.

6. Hoffman M. Tuboovarian abscess in postmenopausal women. J Reprod Med. 1990;35:525-8.

7. Halperin R. Predictors of tuboovarian abscess in acute pelvic inflammatory disease. J Reprod Med. 2008;40-53.

8. Joyeux E. Before surgery predictability of malignant ovarian tumors based on ADNEX model and its use in clinical practice. Gynecol Obstet Fertil. 2016;44(10):557-64.

9. Ognong-Boulemo A, Dohan A, Hoeffel C, Stanek A, Golfier F, Glehen O, Valette PJ, Rousset P. Adnexal masses associated with peritoneal involvement: diagnosis with CT and MRI. Abdominal Radiol. 2017:1-8.

10. Foti PV. MR imaging of ovarian masses: classification and differential diagnosis. Insights Imaging. 2016:7(1):21-41.

11. Kouijzer IJE. Initially unrecognised group A streptococcal pelvic inflammatory disease in a postmenopausal woman. Neth J Med. 2014;72:4946.

12. Mascolino A. Large retroperitoneal abscess extended to the inferior right limb secondary to a perforated ileal Crohn's disease: the importance of the multidisciplinary approach. G Chir. 2016:37:37-41.

13. Zheng J. Gangrenous appendicitis caused huge retroperitoneal abscess and scrotal abscess: a case report and literature review. Int J Clin Exp Med. 2016;9(10):20383-6.

14. Ng NY, Twoon M, Thomson SE. Psoas abscess and severe fasciitis due to a caecal carcinoma. BMJ case reports. 2015;2015:bcr2014207494.

15. Spiliopoulos D. Psoas Abscess after Radical Abdominal Hysterectomy: A Case Report and Review of the Literature. The Open Clin Cancer J. 2010;4:15-9.

16. Christensen L. Necrotizing Fasciltis Case Presentation and Literature Review. N Y State Dent J. 2015;81(4):24-8.

17. Gujral S. Necrotizing Fasciitis. 2014 Available at https://www.ncbi.nlm.nih.gov/pmc/articles/PMC403 4121/pdf/eplasty14ic15.pdf.

18. Hakkarainen TW. Necrotizing soft tissue infections: Review and current concepts in treatment, systems of care, and outcomes. Curr Probl Surg. 2014;51(8):344-62.

19. Amaranathan A. Retroperitoneal Necrotizing Fasciitis Masquerading as Perianal Abscess-Rare and Perilous. Cureus. 2017;9(1):982.

20. Singer M. The Third International Consensus Definitions for Sepsis and Septic Shock (Sepsis-3). JAMA, 2016;315(8):801-10.

21. National Institute for Health and Care Excellence. Sepsis: recognition, assessment and early management; 2016.

22. Lepper PM. Clinical implications of antibioticinduced endotoxin release in septic shock // Intensive Care Medicine. 2002;28:824-33.

23. Holzheimer RG. Antibiotic Induced Endotoxin Release and Clinical Sepsis: a Review. J Antimicrob Chemother. 2013;3:159-72.

24. Otto M. Staphylococcus epidermidis: the accidental pathogen. Nat Rev Microbiol. 2009;7(8):555-67.

25. Almasaudi SB. Acinetobacter spp. as nosocomial pathogens: Epidemiology and resistance features. Saudi J Biologic Sci; 2016.

Cite this article as: Kornete A, Paunina Z, Grabe Z. Complicated clinical course of acute pelvic inflammatory disease. Int J Reprod Contracept Obstet Gynecol 2017;6:4116-9. 